\title{
Narrowband Perfect Absorber Based on Dielectric-Metal Metasurface for Surface-Enhanced Infrared Sensing
}

\author{
Guilian Lan ${ }^{1}$, Zhongxie Jin ${ }^{1, *}$, Jinpeng Nong ${ }^{1}$, Peng Luo ${ }^{1}$, Caicheng Guo ${ }^{1}$, Zhengguo Sang ${ }^{1}$, \\ Lei Dong ${ }^{2}\left(\mathbb{B}\right.$ and Wei Wei ${ }^{1, *} *$ (B)
}

1 Key Laboratory of Optoelectronic Technology \& Systems, Ministry of Education of China, College of Optoelectronic Engineering, Chongqing University, Chongqing 400044, China; 20133092@cqu.edu.cn (G.L.); 20120813077@cqu.edu.cn (J.N.); 20133055@cqu.edu.cn (P.L.); 20152608@cqu.edu.cn (C.G.); zhengry@cqu.edu.cn (Z.S.)

2 State Key Laboratory of Quantum Optics and Quantum Optics Devices, Institute of Laser Spectroscopy, Shanxi University, Taiyuan 030006, China; donglei@sxu.edu.cn

* Correspondence: jinzhongxie@cqu.edu.cn (Z.J.); wwei@cqu.edu.cn (W.W.);

Received: 18 February 2020; Accepted: 19 March 2020; Published: 27 March 2020

\begin{abstract}
We proposed a narrowband perfect absorber that was based on dielectric-metal metasurface for wide-band surface-enhanced infrared sensing. It is found that the narrowband perfect absorber can generate the hybrid guided modes with high quality-factor at infrared frequencies, which make the absorber highly sensitive to the surrounded analyte. Moreover, tuning the incident angle can actively modulate the resonant wavelength of absorber. Such an absorber with excellent features is employed to realize both refractive index sensing and infrared vibrational fingerprint sensing on a single substrate. It is demonstrated that a refractive index sensitivity of $1800 \mathrm{~nm} / \mathrm{RIU}$ and figure of merit of $62 \mathrm{RIU}^{-1}$ can be obtained as the refractive index sensor. While, as a surface enhanced infrared absorption spectroscopy substrate, two closed vibrational modes of analyte with nanometer thick layers can be effectively identified and selectively detected with 50-folds enhancement by actively tuning the incident angle without any change in the structural parameters (periodicity, width, height, and refractive index of the grating) of the device after fabricating. Our study offers a promising approach for designing high-performance surface-enhanced infrared optical sensors in the infrared region.
\end{abstract}

Keywords: surface-enhanced infrared sensing; narrowband perfect absorber; dielectric-metal metasurface

\section{Introduction}

Surface-enhanced infrared optical sensing technology is powerful for direct label-free analysis with high reliability [1,2]. This technology can detect minute refractive index (RI) change of analyte [3-5]; more importantly, it can identify the vibrational mode of target molecules by combining with Fourier Transform Infrared Spectrometer [2,6], which plays an increasingly important role in chemical detection, food safety, and biosensing [7-9]. As a kind of fundamental device for surface-enhanced infrared sensing, plasmonic perfect absorbers based on varying metallic structures have been widely employed, such as Metal-Insulator-Metal (MIM) structure [10-12], Vertical-Square-Split Ring (VSSR) structure [13], Semiconductor-Metal-Semiconductor (SMS) structure [14], Hybrid metasurface [15], and so on. However, the resonance spectra of these absorbers possess full width of half maximum (FWHM) far larger than tens even hundreds of nanometers, owing to the inevitable Ohmic loss of metals, especially when the working wavelength is extended to the important infrared fingerprint 
region [16-18]. This results in a low figure of merit (FOM) and significantly hampers the engineering of infrared sensor with high performance.

Several strategies have been developed to narrow-down the linewidth of resonance spectra, such as adopting gain media to compensate for the losses of metal material $[19,20]$, designing complex nanostructures for modes hybridization $[17,21]$, and synthesizing alternative low-loss plasmonic materials [22,23], to address this obstacle. However, these approaches usually accompany more complex configurations or synthesizing procedures, which greatly limits the practical application of narrowband absorbers in surface-enhanced infrared sensing. To this end, a promising and simple strategy for realizing the narrowband perfect absorbers is to combine guide mode resonance of the dielectric metasurface with the high absorption features of metal [24-26]. In such a strategy, the free loss dielectric metasurface responses for the generation of narrowband guide mode resonance with high quality factor, while the metal can induce a large modulation depth that is beneficial for detecting the minute variations of surrounding media $[19,27]$. These advantages provide a brand-new opportunity for designing the perfect absorber based optical sensors with high FOM. In the visible ranges, by placing the periodical dielectric disk array on metal film, narrowband perfect absorbers have been obtained to detect refractive index change with impressive performance [28,29]. Whereas, in the important infrared region, narrowband perfect absorbers are still lacking for the systematical investigation, especially in infrared vibrational fingerprint sensing, which is complementary to refractive index sensing and allows for us to achieve more information of the analyte $[4,30,31]$.

Inspired by this idea, here we theoretically present a surface-enhanced infrared sensor employing an absorber based on dielectric-metal metasurface. Such an absorber possesses the features of narrowband and angle-dependent resonant absorption, which enable us to perform refractive index sensing and selectively surface-enhanced infrared absorption spectroscopy in one single substrate simultaneously. Specifically, a narrow resonant absorption peak with high quality factor can be obtained due to the loss-free feature of dielectric and high absorption feature of metal. This feature makes the absorber an outstanding refractive index sensor with high sensitivity and FOM. Additionally, the resonant absorption can be actively tuned by adjusting the incident angle, significantly broadening the working waveband of the absorber. This feature makes the absorber an excellent surface-enhanced infrared absorption spectroscopy (SEIRAS) substrate that can selectively detect multi-vibrational modes of molecules in a wide waveband without any change in the structural parameters.

\section{Structure and Methods}

Figure 1 illustrates a schematic view of the proposed surface-enhanced infrared sensor employing angle-tunable narrowband absorber, where a patterned dielectric grating strips array is resting on a thick metal (aluminum) film. The material of the dielectric grating can be $\mathrm{CaF}_{2}$, which has no substrate phonon effect in the considered infrared regime, and the material of the substrate is Si. Here, aluminum is used because the small absorption coefficient that leads to a narrower absorption as compared to gold and silver [32]. As a transverse magnetic (TM) polarized light irradiates upon the metasurface with an angle of $\theta\left(0^{\circ} \leq \theta<90^{\circ}\right)$, dielectric guide mode resonance can be excited at the resonant wavelength, and the energy stored in the excited guided-mode resonance is efficiently absorbed by the metal layer, yielding a peak in the absorption spectra. Such a resonant peak is sensitive to the refractive index change around the dielectric grating. The refractive index change of surrounding analytes would induce a wavelength shift $(\Delta \lambda)$ in the absorption spectrum, which enables us to detect the refractive index of the surrounding analytes, as shown in the schematic diagram in Figure 1. Moreover, this resonant peak also can be tuned (from $\lambda_{1}$ to $\lambda_{3}$ ) to overlap with the molecular vibrational mode by adjusting the incidence angle (from $\theta_{1}$ to $\theta_{3}$ ), so as to selectively identify the vibrational mode of analyte. The combination of these features exhibits great potential application of our device for the infrared refractive index sensing and surface-enhanced infrared absorption spectroscopy in a single substrate. 

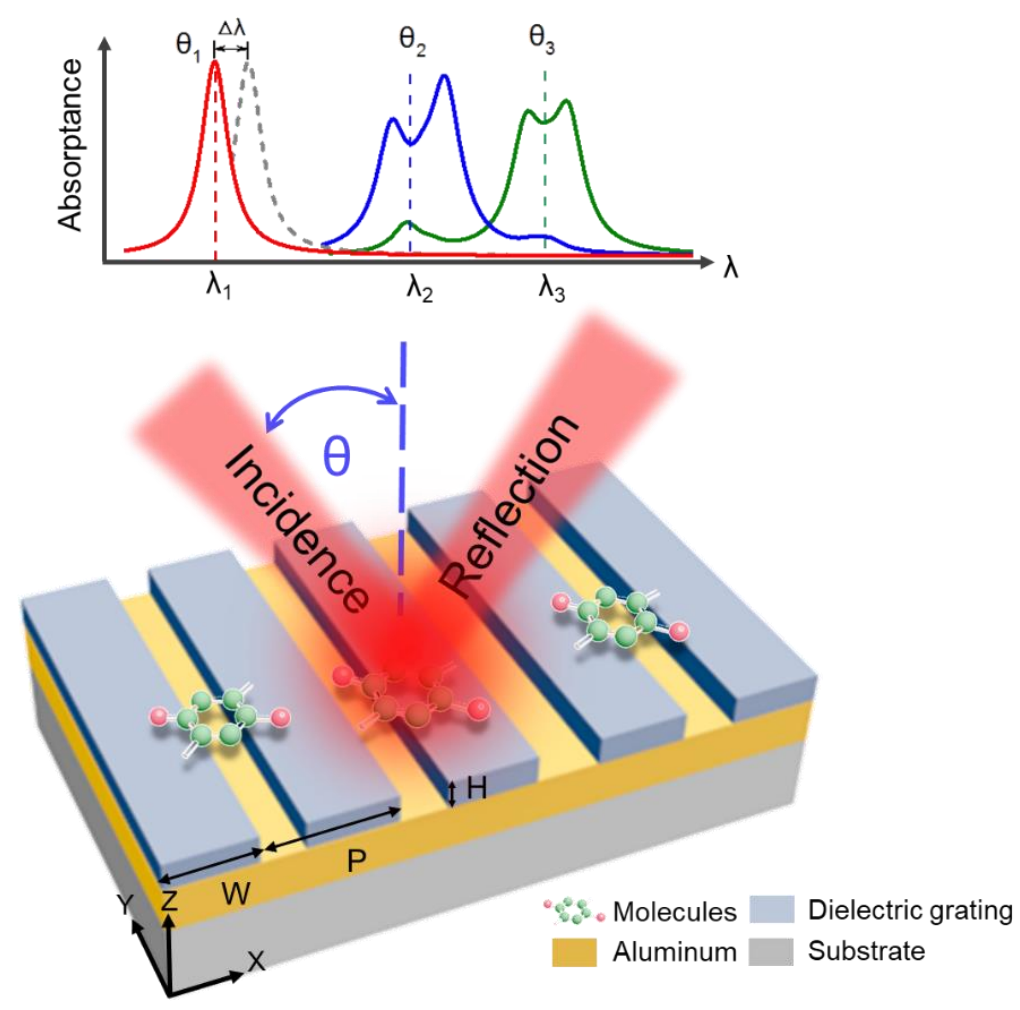

Figure 1. Schematic of the surface-enhanced infrared sensor employing narrowband perfect absorber based on dielectric-metal metasurface.

Simulations are performed employing finite element software COMSOL Multiphysics to investigate the absorption properties of the dielectric-metal metasurface narrowband absorber and its application in RI sensing and SEIRAS. The periodic boundary condition is used in the x-direction, while the perfectly matched layers and scattering boundary conditions are applied in the $\mathrm{z}$ direction at two ends of computational space. The Figure S1 shows the cross-section view of the absorber in COMSOL. A TM polarized light with an electric field intensity of $1 \mathrm{~V} / \mathrm{m}$ vertically irradiates upon the structure. In the simulations, the dielectric grating strip array has a period of $\mathrm{P}$, width of $\mathrm{W}$, height of $\mathrm{H}$, and refractive index of $n_{g}$. The refractive index of aluminum comes from Ref [33].

\section{Results and Discussion}

\subsection{Absorption Properties of the Absorber}

We first investigate the absorption properties of the dielectric-metal metasurface absorber. The absorption (A, red line), and Figure 2a shows the reflection (R, black line) spectra of the absorber with normal incidence. For a thick aluminum plane with thickness larger than the penetration depth of electromagnetic waves in the infrared range, the transmittance of the structure is nearly zero and the absorptance is $A=1-R$. It can be seen that there is a sharp peak in the absorption spectrum with nearly total absorption at the resonant wavelength of $6.134 \mu \mathrm{m}$, which is caused by the inevitable loss of the aluminum and it is known as perfect absorption at the resonant wavelength [34]. The full width half maximum (FWHM) of the spectrum is 29nm with high quality factor [29] $(\mathrm{Q}=\lambda / \Delta \lambda)$ of 212, which can provide impressive performance for infrared optical sensing applications. 

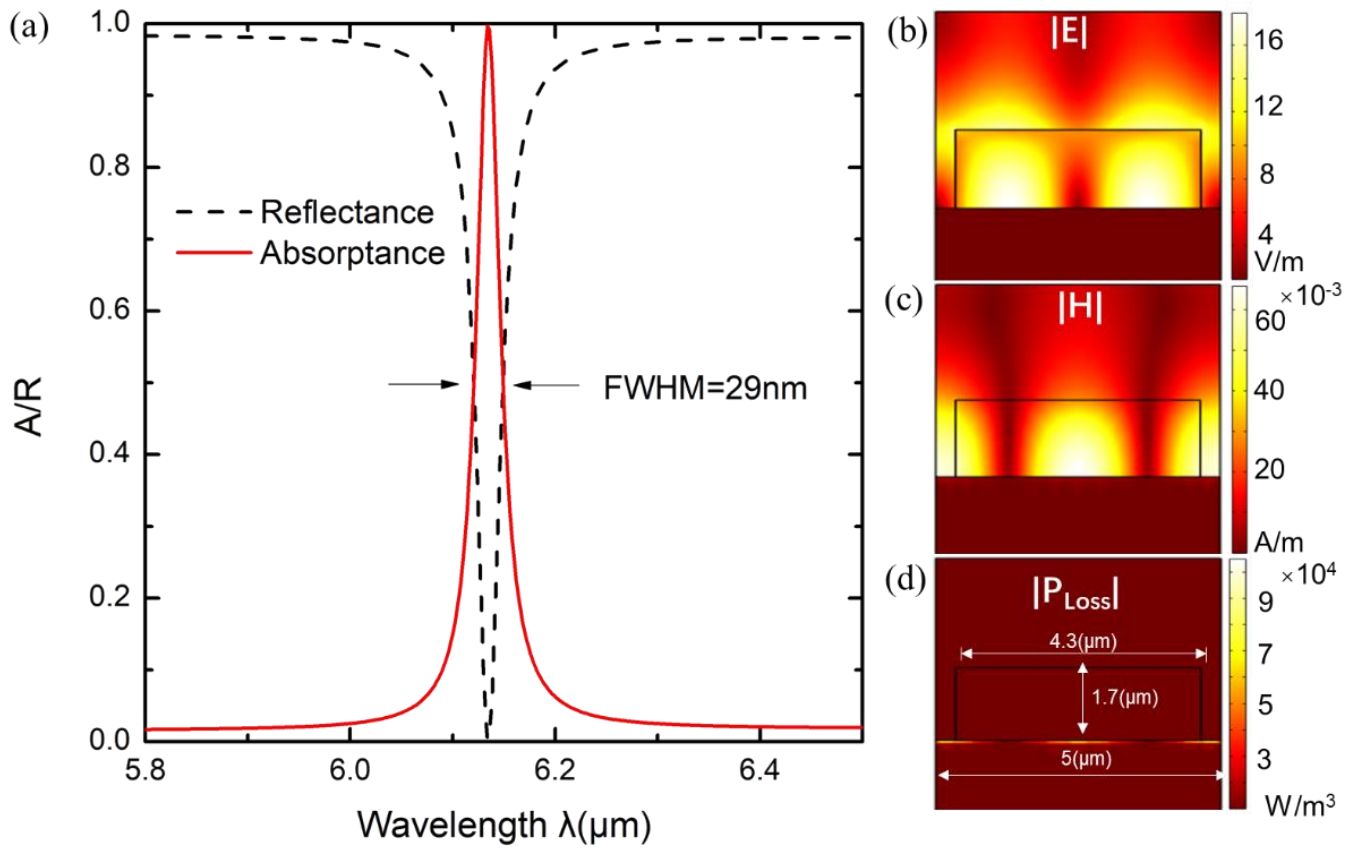

Figure 2. (a) Absorption and reflection spectra of the absorber. The parameters of the dielectric grating strips are $\mathrm{P}=5 \mu \mathrm{m}, \mathrm{W}=4.3 \mu \mathrm{m}, \mathrm{H}=1.7 \mu \mathrm{m}$; (b) Electric field distribution; (c) Magnetic field distribution; and, (d) Power loss distribution of the absorber at resonant wavelength of $6.134 \mu \mathrm{m}$.

We further calculate the mode patterns of electric field, magnetic field, and power loss distribution of the absorber at the resonant wavelength to understand physical mechanism of the narrow linewidth and high absorption of the resonant spectra. Figure $2 b$ obviously shows that one part of electric field is trapped inside the dielectric grating strip, while another part is concentrated at the ends on the topside surface and extended deeply into the surrounding medium. This significantly enhances the light-matter interaction at the dielectric grating strip surface and makes it sensitive to the refractive index change of the surrounding medium. Additionally, the maximum electric field intensity is located at the bottom and top of dielectric grating strip, indicating that the electric dipoles interacting with their images at the dielectric-metal interface. This circular displacement currents lead to the magnetic dipole resonance that is depicted in Figure 2c. The results show that the satisfaction of wavevector matching condition involves the surface lattice resonance mode of the strip array, and the guided mode in the dielectric grating [28]. The power loss pattern in Figure $2 \mathrm{~d}$ shows that the power loss is distributed in the thin domain under the dielectric-metal interface. This can be attributed to the intrinsic loss of metal and the loss-free guided modes in dielectric strip, which results in the narrow linewidth of absorption spectra.

\subsection{RI Sensor Employing High Q-Factor Absorber}

The refractive index of the dielectric grating strips $\left(\mathrm{n}_{\mathrm{g}}\right)$ greatly influences the absorption features of the absorber, since the dielectric grating strips are responsible for the generation of the narrow guide mode resonance. As given in Figure 3a, when the refractive index $\left(\mathrm{n}_{\mathrm{g}}\right)$ increases from 1.4 to 2.2 , the resonant wavelength redshifts from $6.134 \mu \mathrm{m}$ to $8.536 \mu \mathrm{m}$, and the FWHM rapidly increases from 29 $\mathrm{nm}$ to $374 \mathrm{~nm}$, leading to the dramatic decrease of the Q-factor from 212 to only 23 . Moreover, the absorptance of the absorber decreases from 0.99 to 0.33 . These results suggest that the dielectric grating strips with a smaller refractive index $\left(\mathrm{n}_{\mathrm{g}}=1.4\right)$ is more favorable for the optical sensing with a larger Q-factor and absorptance. 

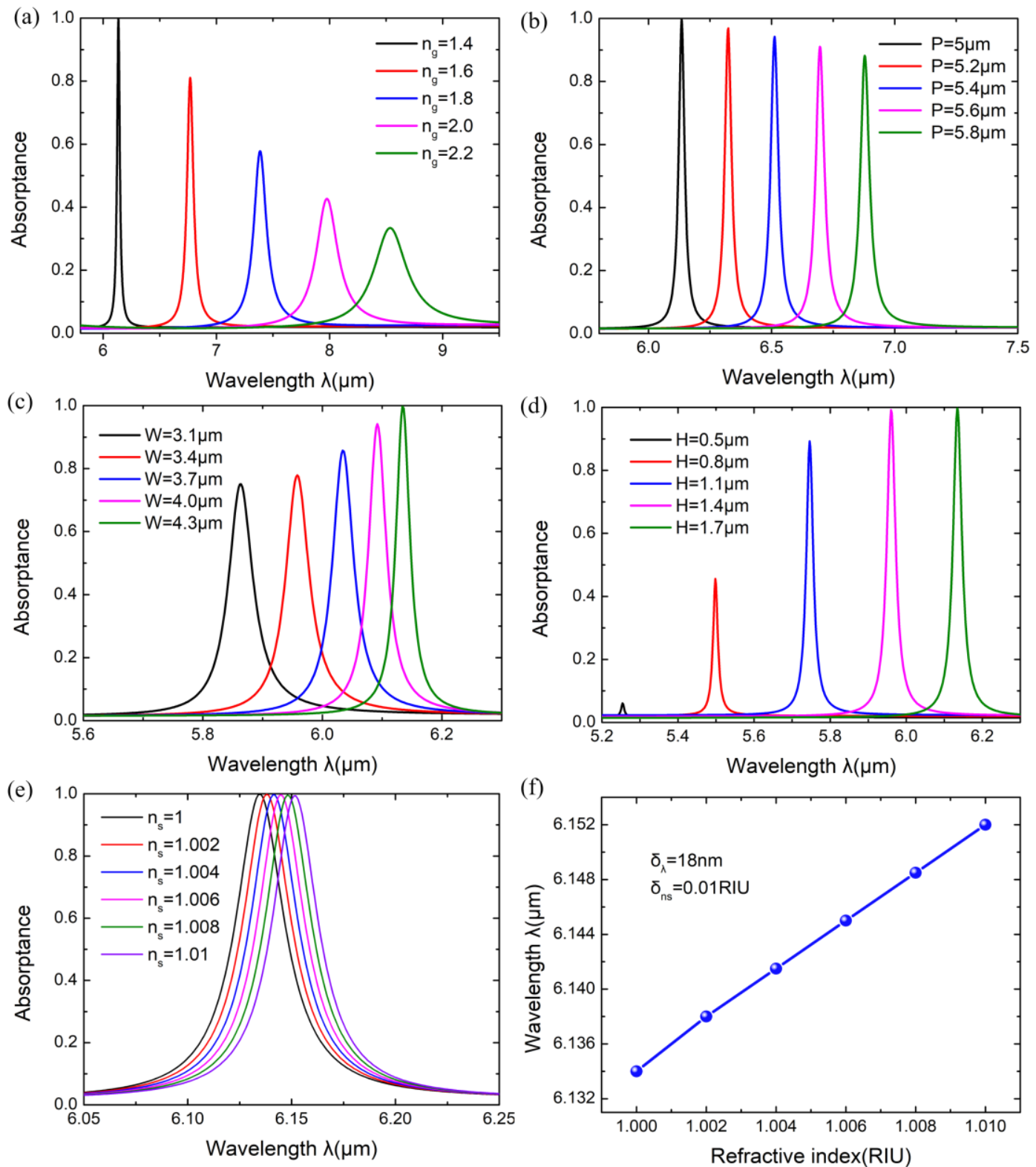

Figure 3. (a) Absorption spectra of the absorber with varying RI $\left(n_{g}\right)$ of dielectric grating strips. (b) Absorption spectra with varying $\mathrm{P}$ when $\mathrm{W}=4.3 \mu \mathrm{m}$ and $\mathrm{H}=1.7 \mu \mathrm{m}$. (c) Absorption spectra with varying $\mathrm{W}$ when $\mathrm{P}=5 \mu \mathrm{m}$ and $\mathrm{H}=1.7 \mu \mathrm{m}$. (d) Absorption spectra with varying $\mathrm{H}$ when $\mathrm{P}=5 \mu \mathrm{m}$ and $\mathrm{W}=4.3 \mu \mathrm{m}$. (e) Absorption spectra with different refractive index of the surrounding environment $\left(\mathrm{n}_{\mathrm{s}}\right)$. (f) Resonant wavelength plotted as a function of refractive index $n_{s}$. The parameters of the dielectric grating strips are $\mathrm{P}=5 \mu \mathrm{m}, \mathrm{W}=4.3 \mu \mathrm{m}, \mathrm{H}=1.7 \mu \mathrm{m}, \mathrm{n}_{\mathrm{g}}=1.4$.

The structural parameters of the dielectric grating strips are further optimized to obtain the high $\mathrm{Q}$-factor absorber. Figure $3 \mathrm{~b}$ illustrates the absorption spectra with varying period $\mathrm{P}$ when $\mathrm{W}=4.3 \mu \mathrm{m}$, $\mathrm{H}=1.7 \mu \mathrm{m}$. One can see that the resonant wavelength exhibits a clear red-shift from $6.134 \mu \mathrm{m}$ to $6.88 \mu \mathrm{m}$ as $P$ increased from $5.0 \mu \mathrm{m}$ to $5.8 \mu \mathrm{m}$. The largest $\mathrm{Q}$-factor of 212 can be achieved for $\mathrm{P}=5.0 \mu \mathrm{m}$. The absorption spectra of the absorber with varying width $\mathrm{W}$ is plotted in Figure 3c. It shows that the resonant wavelength red shift from $5.86 \mu \mathrm{m}$ to $6.134 \mu \mathrm{m}$ and the absorption increase from 0.75 to 0.99 with the increasing of W. Additionally, it is found that the higher Q-factor can be achieved for the larger $\mathrm{W}$, which can be attributed to the increased electromagnetic confinement that is induced by the strong coupling between the dielectric strips. As for the height $\mathrm{H}$ of the dielectric grating strips, it was 
observed that the absorption spectra of the absorber exhibited a red-shift as $\mathrm{H}$ increased from $0.5 \mu \mathrm{m}$ to $1.7 \mu \mathrm{m}$, accompanied with an increased resonant absorption, as shown in Figure 3d. Moreover, the $\mathrm{Q}$-factor is larger for the larger height of the grating. These results indicate that the spectral features of absorber can be tuned by the structural parameters of dielectric strips to achieve a high Q-factor value. Additionally, the absorption spectra with resonant wavelength located in lower fingerprint regime are given in Figure S2. For the experimentalists who want to tune the resonance wavelength to another spectral regime, he (she) can first chose a grating with a proper period to locate the resonant wavelength into the interested spectral regime, and then optimize the width and height of the grating to obtain the devices with high absorption and Q-factor.

The high Q-factor narrowband absorber is firstly employed as a high-performance refractive index sensor in the infrared region. From the electric field distributions depicted in Figure 2b, we can find that the electric field is concentrated on the topside surface ends of the dielectric grating strips, which can be easily accessible to the surrounding analytes and makes it very sensitive to the refractive index change that is induced by the change of the analytes concentration. Such a change in refractive index can be measured by detecting the wavelength shift of the resonant peak. Therefore, the presented metasurface absorber has desirable spectral features for refractive index sensing applications in the infrared region. To evaluate the performance of the sensor, the sensitivity (S) and the Figure of merit (FOM) are defined, as following [16]

$$
\begin{aligned}
S & =\frac{\Delta \lambda}{\Delta n} \\
F O M & =\frac{S}{F W H M}
\end{aligned}
$$

$\Delta \lambda$ is the shift of resonant wavelength due to change $\Delta \mathrm{n}$ of RI in the surrounding. The resonant wavelength has a redshift of $18 \mathrm{~nm}$ when the refractive index of the surrounding analytes $\left(n_{\mathrm{s}}\right)$ changes from 1 to 1.01, as shown in Figure 3e. The corresponding resonant wavelength was further extracted and plotted as a function of the refractive index in Figure 3f. The RI sensitivity and the FOM are estimated to be $S=1800 \mathrm{~nm} / \mathrm{RIU}$ and FOM $=62 \mathrm{RIU}^{-1}$ for the proposed metasurface absorber with Q-factor of 212, where RIU is Refractive Index Unit and RIU ${ }^{-1}$ is unit of the FOM, according to Equations (1) and (2). The refractive index sensing performance of this absorber is compared with that of the other kinds of absorber with different structure reported recently, as summarized in Table S1. It can be seen that the sensitivity and FOM of proposed absorber are comparable to or even larger than those of plasmonic sensing devices (such as MIM structure, VSSR Resonator and Hybrid metasurface). When considering that the optic spectrometer has resolution of $1 \mathrm{~nm}$, the detection limit of the sensor is $5.6 \times 10^{-4} \mathrm{RIU}$, which suggests that the proposed refractive index sensor employing high Q-factor absorber offers a promising platform for surface-enhanced infrared sensing applications.

\subsection{SEIRAS Substrate Employing Angle-Tunable Absorber}

Besides the refractive index sensing, the narrowband perfect absorber with high electromagnetic field confinement can also be used as the surface-enhanced infrared absorption spectroscopy substrate to identify the target molecules, which is complementary to refractive index sensing, since it can detect molecular vibrational modes. It is worth noting that the absorption spectrum of the present absorber can be actively tuned by the incident angle in a wide waveband, as shown in the absorptance map at oblique incidence in Figure $4 \mathrm{a}$. The absorptance spectra with varying incident angle when $\theta=0 \mathrm{deg}$, $\theta=5 \mathrm{deg}$, and $\theta=10 \mathrm{deg}$ are further extracted from Figure $4 \mathrm{a}$ and plotted in Figure $4 \mathrm{~b}$. We can see that the resonance peak splits into two individual peaks and shift towards the opposite directions as the incident angle increases. One of the resonant peak blue shifts to $5.25 \mu \mathrm{m}$ and the other one red shift to $6.80 \mu \mathrm{m}$ when $\theta=10 \mathrm{deg}$, which significantly broadens the working waveband of the absorber for SEIRAS. 

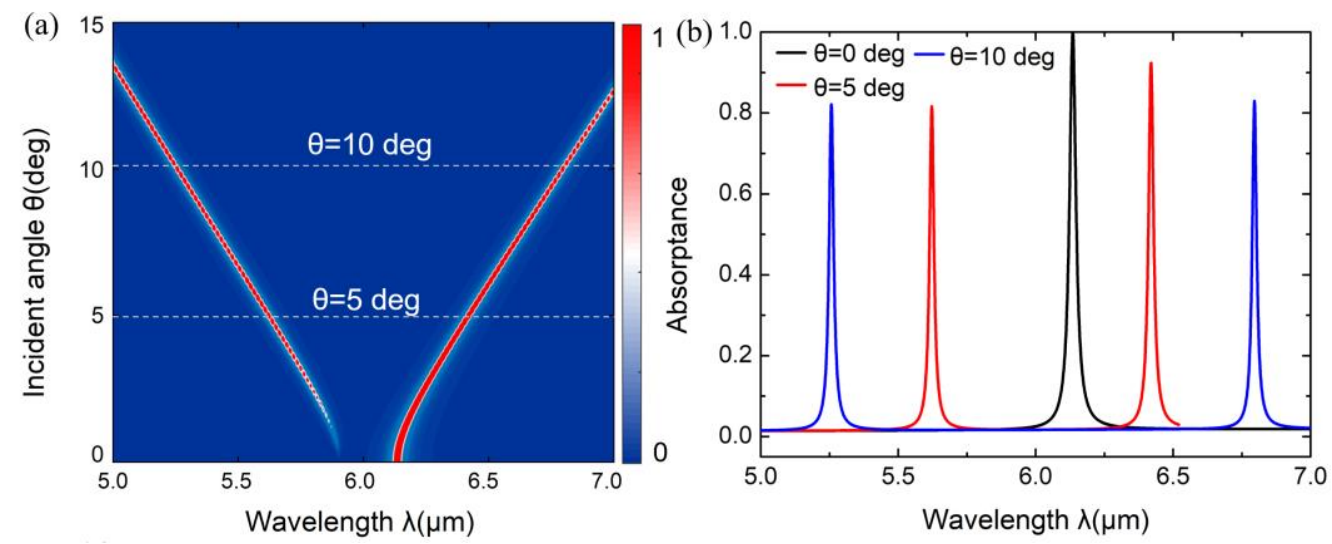

Figure 4. (a) Absorptance map of the dielectric-metal metasurface absorber with different incident angle. (b) The absorptance spectra with varying incident angle when $\theta=0 \mathrm{deg}, \theta=5 \mathrm{deg}$ and $\theta=10 \mathrm{deg}$.

We then considered the electromagnetic coupling between the guide modes and infrared vibrational modes of molecules to explore the performance of the proposed SEIRAS substrate. The dielectric constant of the molecules can be described as the Drude-Lorentz type dispersion $[35,36]$

$$
\varepsilon(\omega)=1+\sum_{n=1}^{N} \frac{e^{2} N_{n} /\left(\varepsilon_{0} m_{0}\right)}{\omega_{n}^{2}-\omega^{2}-i \gamma_{n} \omega}
$$

where $m_{0}$ is the electron rest mass, $\omega_{n}$ is the molecular resonant frequency, $N_{n}$ is the volume density of molecular dipoles, and $\gamma_{\mathrm{n}}$ is the molecular damping. Here, we start by considering a $10 \mathrm{~nm}$-thick molecular film as the analyte with one single vibrational mode located at $6.225 \mu \mathrm{m}$, corresponding to vibrational mode of aromatic ring that is commonly seen in many organic matter, such as perylene-3,4,9,10-tetracarboxylic dianhydride (PTCDA) [37]. Its dielectric constant that is calculated by Equation (3) is plotted in Figure $5 \mathrm{a}$. Figure $5 \mathrm{~b}$ presents the absorptance spectra of absorber covered with (dashed line) and without (solid line) a molecular film for TM polarization light with $\theta=2 \mathrm{deg}$, respectively. An enlarged (10-fold) absorption spectrum of this molecular vibrational mode is also plotted (blue line). One can see that the direct absorption of the molecule vibrational mode is low (only $0.54 \%$ ), which is attributed to the weak interaction between incident infrared light and the molecules. When the molecules are adsorbed on the absorber surface, the weak vibrational modes are significantly enhanced, which feature strong dips over the resonance peaks. 

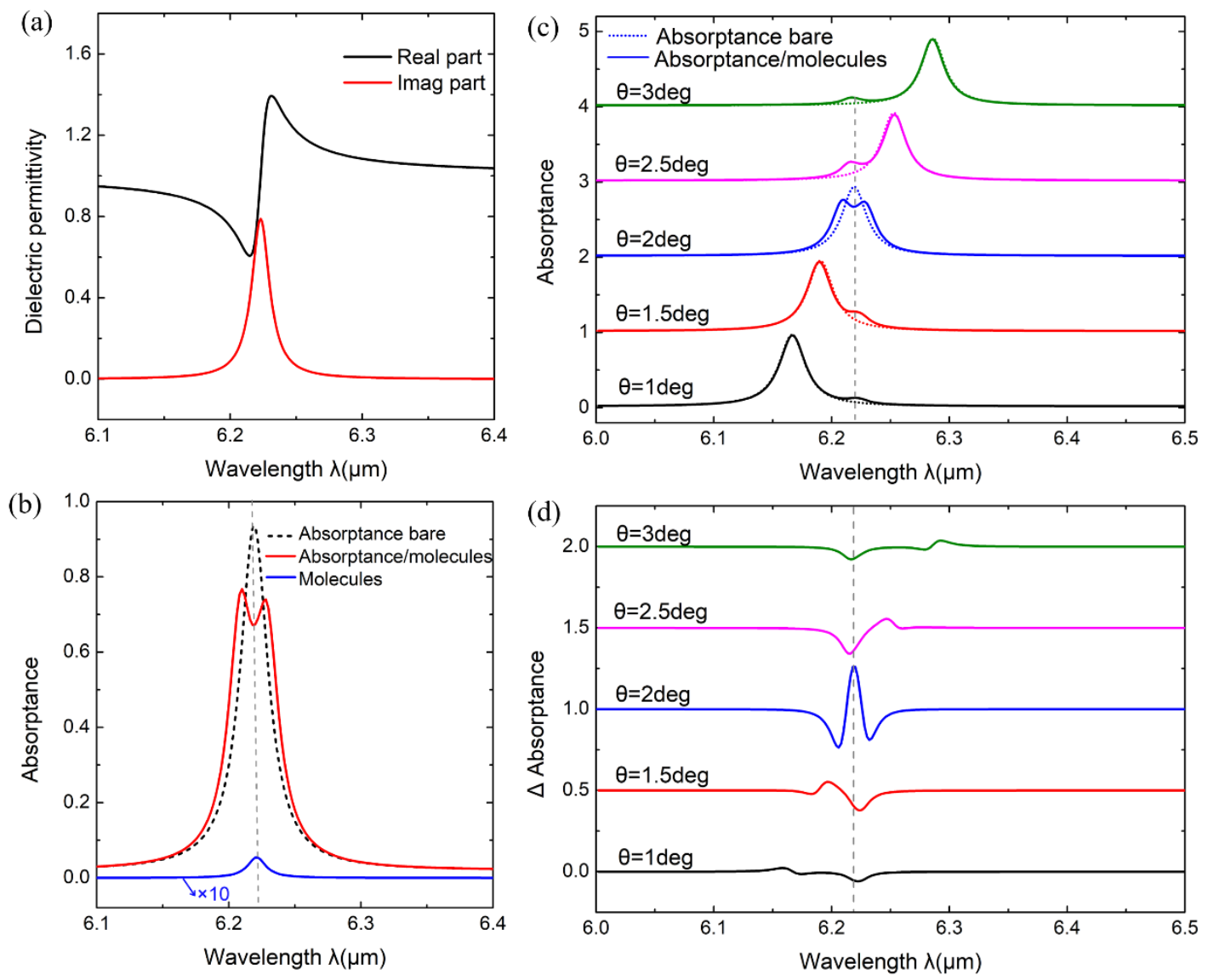

Figure 5. (a) The dielectric permittivity of the molecular film with one vibrational mode at $6.225 \mu \mathrm{m}$ when $\mathrm{N}_{\mathrm{n}}=0.1 \mathrm{~mol} \mathrm{~m}{ }^{-3}, \gamma_{\mathrm{n}}=8 \times 10^{11} \mathrm{rad} / \mathrm{s}$. (b) Absorption spectra of the absorber covered with (solid line) and without (dashed line) 10-nm-thick molecules layer for transverse magnetic (TM)-polarized light with $\theta=2$ deg. (c) Absorption spectra of absorber covered with and without molecules in different incident angle. (d) Delta absorptance of absorber covered with molecules in different incident angle.

The resonant wavelength of absorber is further dynamically tuned by the incident angle, so that it can overlap with the molecule vibrational mode to achieve the maximum enhancement. Figure $5 \mathrm{c}$ shows the absorption spectra of absorber before (dashed lines) and after (solid lines) being adsorbed by the molecules film with varying incident angle. As expected, the enhanced signals vary in strength and line shape, depending on their spectral position relative to the vibrational mode of the molecular film and the dielectric guide mode of the absorber when these two modes are overlapped. Figure $5 \mathrm{~d}$ stacks the corresponding enhanced vibrational signals (delta absorptance) of molecules film. Obviously, the vibrational signal that is close to the dielectric guide mode resonance peak much larger amplitude than those wing signals. The maximum signal strength of vibrational mode is $26.8 \%$ when the incident angle is $2 \mathrm{deg}$, which is about 50 times with respect to the intrinsic absorption of the molecule film without resonance enhancement. Additionally, this value is comparable to even larger than the enhancement of some reported plasmonic sensors [38,39]. We further investigate the absorption spectra with different thickness of molecules layer to evaluate the confinement, as shown in Figure S3. The enhanced signal strength increases significantly when the thickness is smaller than $80 \mathrm{~nm}$, whereas the signal strength is hardly increased after the thickness is larger than $80 \mathrm{~nm}$, indicating that the electromagnetic energy is mainly confined within $80 \mathrm{~nm}$ from the dielectric surface.

The angle-tunable property of the absorber makes it possible to sense the molecular films with two or even more vibration modes while using the same substrate in the wide waveband. We further consider the two chosen individual vibration modes of PTCDA film located at $6.225 \mu \mathrm{m}$ and $6.355 \mu \mathrm{m}$ 
to illustrate the frequency-selective enhancement of molecular vibrational modes (labeled as A mode and B mode shown in Figure 6a). The absorptance of the absorber covering the molecules is mapping as a function of wavelength and incident angle in Figure 6b. It is intuitively observed that the interaction between the molecular vibrational modes and the dielectric guide mode near-field leads to a splitting of the infrared absorption spectra at the position of the molecular absorption lines, which significantly enhances the molecular absorption of $A$ and $B$ modes. This is due to the large energy exchange between the dielectric hybrid guide modes and the molecular vibrational modes when they are spectrally matched by tuning the incident angle [40].
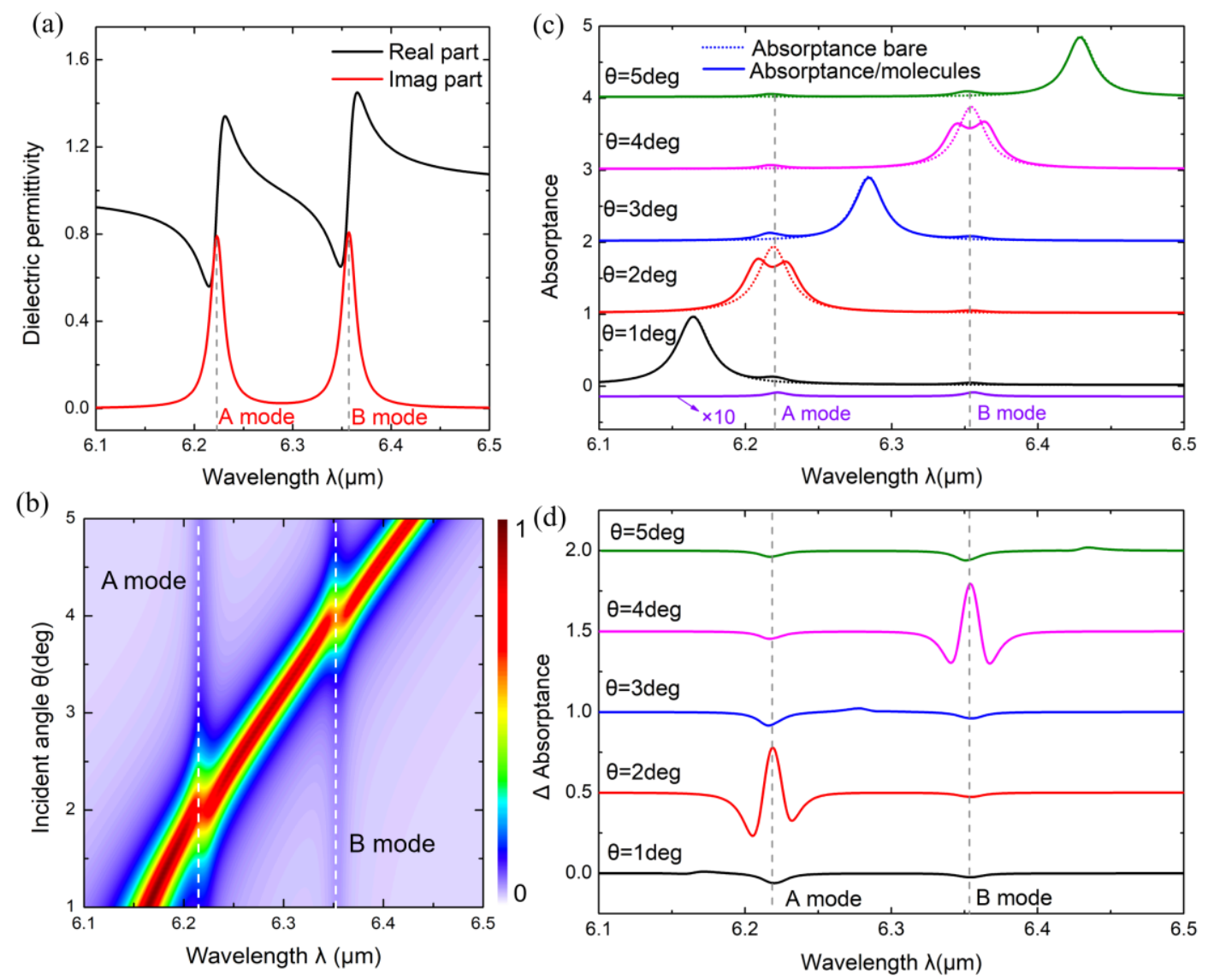

Figure 6. (a) The dielectric permittivity of the molecular film with two vibrational modes at $6.225 \mu \mathrm{m}$ and $6.355 \mu \mathrm{m}$ when $\mathrm{N}_{\mathrm{A}}=\mathrm{N}_{\mathrm{B}}=0.1 \mathrm{~mol} \mathrm{~m}{ }^{-3}, \gamma_{\mathrm{A}}=\gamma_{\mathrm{B}}=8 \times 10^{11} \mathrm{rad} / \mathrm{s}$. (b) Absorptance map of the dielectric-metal absorber covered with molecules film with different incident angle. (c) Absorption spectra of absorber covered with and without molecules film in different incident angle. (d) Delta absorptance of absorber covered with molecules film in different incident angle.

To be more specific, the absorption spectra of the absorber covered with the molecules film having two vibrational modes are further extracted from Figure $6 \mathrm{~b}$ and illustrated in Figure $6 c$. It can be seen that the resonant peak exhibits a strong dip at the A mode when the incident angle is 2 deg, while it exhibits a strong dip at the B mode with the $\theta=4 \mathrm{deg}$. The delta absorptances of the two vibrational modes are calculated to further quantitatively evaluate the signal enhancement, as shown in Figure 6d. It is obvious that the maximal differential absorption of A mode at $6.225 \mu \mathrm{m}$ is $26.8 \%$ at the incident angle of $2 \mathrm{deg}$, thus corresponding to the enhancement of 50-folds. Subsequently, it decreases to $3.03 \%$ when the angle increases from $2 \mathrm{deg}$ to $4 \mathrm{deg}$, which is expected since the guide mode resonant wavelength is tuned away from this mode. However, the signal strength of $B$ mode increases rapidly since the guide mode resonant wavelength is tuned towards B mode at $6.355 \mu \mathrm{m}$. At $\theta=4 \mathrm{deg}$, the guide mode resonance is approximately adjusted to the B mode, giving the large signal 
strength of $26 \%$. Therefore, two even more vibrational modes of molecules can be selectively enhanced in a wide waveband by actively tuning the incident angle.

\section{Conclusions}

In summary, we proposed a surface-enhanced infrared sensor employing a narrowband perfect absorber based on dielectric-metal metasurface, where a dielectric grating array is resting on an aluminum plane. The excellent features of the narrowband perfect absorber allow for us to realize both refractive index sensing and SEIRAS on a single substrate in the infrared region. It is found that the high sensitivity of $1800 \mathrm{~nm} / \mathrm{RIU}$ and FOM of $62 \mathrm{RIU}^{-1}$ can be obtained when the absorber as a refractive index sensor due to the high Q-factor of the perfect absorber. More importantly, it is used as a SEIRAS substrate to enhance the molecular vibration mode with the high enhancement of 50 -folds when the hybrid guided modes of absorber and the vibrational mode of molecules are spectrally matched. Finally, the working waveband of the absorber is significantly broadened by tuning the incident angle, which provides a feasible way to selectively detect multi vibrational modes of molecules in broad range without any change in the parameters of periodicity, width, height, and refractive index of the grating after the device was processed. Our study shows that the dielectric-metal metasurface perfect absorber has promising potential in the design of surface-enhanced infrared sensors.

Supplementary Materials: The following are available online at http://www.mdpi.com/2076-3417/10/7/2295/s1, Figure S1: The cross-section view of the proposed nanostructure, Figure S2: (a) Absorptance of the metasurface absorber with different height $\mathrm{H}$ of the dielectric grating strips when $\mathrm{P}=3 \mu \mathrm{m}$ and $\mathrm{W}=2.3 \mu \mathrm{m}$; (b) Corresponding absorptance and Q-factor with different $\mathrm{H}$ of the dielectric grating strips. (c) Absorption spectra of the metasurface absorber with different groove width $\mathrm{W}$ when $\mathrm{P}=3 \mu \mathrm{m}$ and $\mathrm{H}=0.7 \mu \mathrm{m} ;(\mathrm{d})$ Corresponding absorptance and $\mathrm{Q}$-factor with different groove width W. (e) Absorption spectra of the metasurface absorber with different period $\mathrm{P}$ when $\mathrm{W}=0.7 \mu \mathrm{m}$ and $\mathrm{H}=0.7 \mu \mathrm{m}$; (f) Corresponding absorptance and Q-factor with different period P, Figure S3: (a) Delta absorptance of absorber covered with molecules layer of different thickness. (b) Enhanced signal strength with different thickness, Table S1: The refractive index sensing performance of the proposed absorber relative to those reported in literatures.

Author Contributions: Formal analysis, G.L.; Writing-original draft, G.L.; Data curation, Z.J.; Investigation, J.N. and P.L.; Software, J.N. and C.G.; Methodology, Z.S. and L.D.; Supervision, Z.J. and W.W.; Writing - review \& editing, W.W. All authors have read and agreed to the published version of the manuscript.

Funding: This research was funded by National Natural Science Foundation of China (No. 61875025, 61675037), Chongqing Research Program of Basic Research and Frontier Technology (cstc2017jcyjBX0048), and Fundamental Research Funds for the Central Universities (2018CDQYGD0022, cqu2018CDHB1B03), Chongqing basic science and frontier technology research project(cstc2017jcyjAX0237), and the Visiting Scholar Foundation of Key lab of Optoelectronic Technology \& Systems(Chongqing University),Ministry of Education. China.

Conflicts of Interest: The authors declare no conflict of interest.

\section{References}

1. Haas, J.; Mizaikoff, B. Advances in mid-infrared spectroscopy for chemical analysis. Ann. Rev. Anal. Chem. 2016, 9, 45-68. [CrossRef] [PubMed]

2. Guo, X.; Hu, H.; Liao, B.; Zhu, X.; Yang, X.; Dai, Q. Perfect-absorption graphene metamaterials for surface-enhanced molecular fingerprint spectroscopy. Nanotechnology 2018, 29, 184004. [CrossRef] [PubMed]

3. Lu, X.; Zhang, T.; Wan, R.; Xu, Y.; Zhao, C.; Guo, S. Numerical investigation of narrowband infrared absorber and sensor based on dielectric-metal metasurface. Opt. Express 2018, 26, 10179-10187. [CrossRef] [PubMed]

4. Imogen, M.; Pryce, Y.A.K.; Koray, A.; Harry, A.A. Compliant metamaterials for resonantly enhanced infrared absorption spectroscopy and refractive index sensing. ACS Nano 2011, 5, 8167-8174.

5. Wei, W.; Nong, J.; Tang, L.; Zhang, G.; Yang, J.; Luo, W. Conformal Graphene-Decorated Nanofluidic Sensors Based on Surface Plasmons at Infrared Frequencies. Sensors 2016, 16, 899. [CrossRef]

6. Hu, H.; Yang, X.; Zhai, F.; Hu, D.; Liu, R.; Liu, K.; Sun, Z.; Dai, Q. Far-field nanoscale infrared spectroscopy of vibrational fingerprints of molecules with graphene plasmons. Nat. Commun. 2016, 7, 12334. [CrossRef]

7. Sharpe, S.J.; Sams, R.L.; Chu, P.M.; Rhoderick, G.C.; Johnson, P.A. Gas-phase databases for quantitative infrared spectroscopy. Appl. Spectrosc. 2004, 58, 1452-1461. [CrossRef] 
8. Wei, W.; Nong, J.; Lan, G.; Wang, W.; Yi, J. Graphene-assisted multilayer structure employing hybrid surface plasmon and magnetic plasmon for surface-enhanced vibrational spectroscopy. Opt. Express 2018, 26, 16903-16916. [CrossRef]

9. Wu, H.; Dong, L.; Yin, X.; Sampaolo, A.; Patimisco, P.; Ma, W.; Zhang, L.; Yin, W.; Xiao, L.; Spagnolo, V.; et al. Atmospheric $\mathrm{CH}_{4}$ measurement near a landfill using an ICL-based QEPAS sensor with V-T relaxation self-calibration. Sens. Actuators B Chem. 2019, 297, 126753. [CrossRef]

10. Cui, W.; Peng, W.; Yu, L.; Luo, X.; Gao, H.; Chu, S.; Masson, J.F. Hybrid Nanodisk film for ultra-narrowband filtering, near-perfect absorption and wide range sensing. Nanomaterials 2019, 9, 334. [CrossRef]

11. Dao, T.; Chen, K.; Ishii, S.; Ohi, A.; Nabatame, T.; Kitajima, M.; Nagao, T. Infrared Perfect Absorbers Fabricated by Colloidal Mask Etching of $\mathrm{Al}_{-} \mathrm{Al}_{2} \mathrm{O}_{3}-\mathrm{Al}$ Trilayers. ACS Photonics. 2015, 2, 964-970. [CrossRef]

12. Tittl, A.; Michel, A.; Schaferling, M.; Yin, X.; Gholipour, B.; Cui, L.; Wuttig, M.; Taubner, T.; Neubrech, F.; Giessen, H. A Switchable Mid-Infrared Plasmonic Perfect Absorber with Multispectral Thermal Imaging. Capab. Adv. Mater. 2015, 27, 4597-4603. [CrossRef] [PubMed]

13. Cheng, Y.; Luo, H.; Chen, F.; Gong, R. Triple narrow-band plasmonic perfect absorber for refractive index sensing applications of optical frequency. OSA Contin. 2019, 2, 2113. [CrossRef]

14. Li, Y.; Liu, Y.; Liu, Z.; Tang, Q.; Shi, L.; Chen, Q.; Du, G.; Wu, B.; Liu, G.; Li, L. Grating-assisted ultra-narrow multispectral plasmonic resonances for sensing application. Appl. Phys. Express 2019, 12, 072002. [CrossRef]

15. Rifat, A.A.; Rahmani, M.; Xu, L.; Miroshnichenko, A.E. Hybrid metasurface based tunable near-perfect absorber and plasmonic sensor. Materials 2018, 11, 1091. [CrossRef]

16. Lu, X.; Wan, R.; Zhang, T. Metal-dielectric-metal based narrow band absorber for sensing applications. Opt. Express 2015, 23, 29842-29847. [CrossRef]

17. Lu, X.; Zhang, L.; Zhang, T. Nanoslit-microcavity-based narrow band absorber for sensing applications. Opt. Express 2015, 23, 20715-20720. [CrossRef]

18. Zhang, B.Z.; Hao, Y.; Kiraly, Q.; Khoo, B.; Chen, I.A.; Huang, S.; Jun, T. Polarization-independent dual-band infrared perfect absorber based on a metal-dielectric-metal elliptical nanodisk array. Opt. Express 2011, 19, 15221-15228. [CrossRef]

19. Xiao, S.; Drachev, V.P.; Kildishev, A.V.; Ni, X.; Chettiar, U.K.; Yuan, H.K.; Shalaev, V.M. Loss-free and active optical negative-index metamaterials. Nature 2010, 466, 735-738. [CrossRef]

20. Shalaev, V.M. Optical negative-index metamaterials. Nat. Photon. 2007, 1, 41-48. [CrossRef]

21. Chanda, D.; Shigeta, K.; Truong, T.; Lui, E.; Mihi, A.; Schulmerich, M.; Braun, P.V.; Bhargava, R.; Rogers, J.A. Coupling of plasmonic and optical cavity modes in quasi-three-dimensional plasmonic crystals. Nat. Commun. 2011, 2, 479. [CrossRef] [PubMed]

22. Naik, G.V.; Shalaev, V.M.; Boltasseva, A. Alternative plasmonic materials: Beyond gold and silver. Adv. Mater. 2013, 25, 3264-3294. [CrossRef] [PubMed]

23. West, P.R.; Ishii, S.; Naik, G.V.; Emani, N.K.; Shalaev, V.M.; Boltasseva, A. Searching for better plasmonic materials. Laser Photon. Rev. 2010, 4, 795-808. [CrossRef]

24. Semouchkina, E.; Duan, R.; Semouchkin, G.; Pandey, R. Sensing based on Fano-type resonance response of all-dielectric metamaterials. Sensors 2015, 15, 9344-9359. [CrossRef] [PubMed]

25. Zhang, J.; Zhu, Z.; Yuan, X.; Qi, S. Strong field enhancement and light-matter interactions with all-dielectric metamaterials based on split bar resonators. Opt. Express 2014, 22, 30889-30898. [CrossRef] [PubMed]

26. Jahani, S.; Jacob, Z. All-dielectric metamaterials. Nat. Nanotechnol. 2016, 11, 23-36. [CrossRef] [PubMed]

27. Artemios, K.B.G.; MacDonald, K.F.; Zheludev, N.I. All-dielectric phase-change reconfigurable metasurface. Appl. Phys. Lett. 2016, 109, 1-5.

28. Liao, Y.-L.; Zhao, Y.; Zhang, X.; Chen, Z. An ultra-narrowband absorber with a compound dielectric grating and metal substrate. Opt. Commun. 2017, 385, 172-176. [CrossRef]

29. Callewaert, F.; Chen, S.; Butun, S.; Aydin, K. Narrow band absorber based on a dielectric nanodisk array on silver film. J. Opt. 2016, 18, 075006. [CrossRef]

30. Wu, H.; Dong, L.; Zheng, H.; Yu, Y.; Ma, W.; Zhang, L.; Yin, W.; Xiao, L.; Jia, S.; Tittel, F. Beat frequency quartz-enhanced photoacoustic spectroscopy for fast and calibration-free continuous trace-gas monitoring. Nat. Commun. 2017, 8, 15331. [CrossRef]

31. Yin, X.; Wu, H.; Dong, L.; Li, B.; Ma, W.; Zhang, L.; Yin, W.; Xiao, L.; Jia, S.; Tittel, F.K. PPb-Level SO 2 Photoacoustic Sensors with a Suppressed Absorption-Desorption Effect by Using a 7.41 mum External-Cavity Quantum Cascade Laser. ACS Sens. 2020, 5, 549-556. [CrossRef] [PubMed] 
32. Maharana, P.K.; Srivastava, T.; Jha, R. On the performance of highly sensitive and accurate graphene-on-aluminum and silicon-based SPR biosensor for visible and near infrared. Plasmonics 2014, 9, 1113-1120. [CrossRef]

33. Rakic, A.D. Algorithm for the determination of intrinsic optical constants of metal films: Application to aluminum. Appl. Opt. 1995, 34, 4755-4767. [CrossRef] [PubMed]

34. Liu, N.; Mesch, M.; Weiss, T.; Hentschel, M.; Giessen, H. Infrared perfect absorber and its application as plasmonic sensor. Nano Lett. 2010, 10, 2342-2348. [CrossRef] [PubMed]

35. Wei, W.; Jiang, X.; Chen, N.; Luo, S.; Tang, L. All-semiconductor plasmonic resonator for surface-enhanced infrared absorption spectroscopy. Micromachines 2017, 8, 6. [CrossRef]

36. Liu, F.; Cubukcu, E. Tunable omnidirectional strong light-matter interactions mediated by graphene surface plasmons. Phys. Rev. B 2013, 88, 115439. [CrossRef]

37. Kelly Akers, R.A. Molecular organization in perylenetetracarboxylic dianhydride films. J. Phys. Chem. 1987, 91, 2954-2959. [CrossRef]

38. Wang, T.; Dong, Z.; Koay, E.H.H.; Yang, J.K.W. Surface-enhanced infrared absorption spectroscopy using charge transfer plasmons. ACS Photon. 2019, 6, 1272-1278. [CrossRef]

39. Wang, C.T.; Jiang, B.; Zhou, Y.W.; Jiang, T.W.; Liu, J.H.; Zhu, G.D.; Cai, W.B. Exploiting the surface-enhanced IR absorption effect in the photothermally induced resonance AFM-IR technique toward nanoscale chemical analysis. Anal. Chem. 2019, 91, 10541-10548. [CrossRef]

40. Nong, J.; Wei, W.; Wang, W.; Lan, G.; Shang, Z.; Yi, J.; Tang, L. Strong coherent coupling between graphene surface plasmons and anisotropic black phosphorus localized surface plasmons. Opt. Express 2018, 26, 1633-1644. [CrossRef]

(C) 2020 by the authors. Licensee MDPI, Basel, Switzerland. This article is an open access article distributed under the terms and conditions of the Creative Commons Attribution (CC BY) license (http://creativecommons.org/licenses/by/4.0/). 